\title{
Argon-Ion Contamination of the Plasmasphere
}

\author{
Prepared by \\ Y. T. CHIU, J. M. CORNWALL, Consultant, J. G. LUHMANN, \\ and MICHAEL SCHULZ \\ Space Sciences Laboratory
}

15 July 1979

Prepared for

ARGONNE NATIONAL LABORATORIES

Argonne, Illinois 60439

Contract No. 31-109-38-5075

Laboratory Operations 


\section{DISCLAIMER}

This report was prepared as an account of work sponsored by an agency of the United States Government. Neither the United States Government nor any agency Thereof, nor any of their employees, makes any warranty, express or implied, or assumes any legal liability or responsibility for the accuracy, completeness, or usefulness of any information, apparatus, product, or process disclosed, or represents that its use would not infringe privately owned rights. Reference herein to any specific commercial product, process, or service by trade name, trademark, manufacturer, or otherwise does not necessarily constitute or imply its endorsement, recommendation, or favoring by the United States Government or any agency thereof. The views and opinions of authors expressed herein do not necessarily state or reflect those of the United States Government or any agency thereof. 


\section{DISCLAIMER}

Portions of this document may be illegible in electronic image products. Images are produced from the best available original document. 


\section{LABORATORY OPERATIONS}

The Laboratory Operations of The Aerospace Corporation is conducting experimental and theoretical investigations necessary for the evaluation and application of scientific advances to new military concepts and systems. Versatility and flexibility have been developed to a high degree by the laboratory personnel in dealing with the many problems encountered in the nation's rapidly developing space and missile systems. Expertise in the latest scientific developments is vital to the accomplishment of tasks related to these problems. The laboratories that contribute to this research are:

Aerophysics Laboratory: Launch and reentry aerodynamics, heat transfer, reentry physics, chemical kinetics, structural mechanics, flight dynamics, atmospheric pollution, and high-power gas lasers.

Chemistry and Physics Laboratory: Atmospheric reactions and atmospheric optics, chemical reactions in polluted atmospheres, chemical reactions of excited species in rocket plumes, chemical thermodynamics, plasma and laser-induced reactions, laser chemistry, propulsion chemistry, space vacuum and radiation effects on materials, lubrication and surface phenomena, photosensitive.materials and sensors, high precision laser ranging, and the application of physics and chemistry to problems of law enforcement and biomedicine.

Electronics Research Laboratory: Electromagnetic theory, devices, and propagation phenomena, including plasma electromagnetics; quantum electronics, lasers, and electro-optics; communication sciences, applied electronics, semiconducting, superconducting, and crystal device physics, optical and acoustical imaging; atmospheric pollution; millimeter wave and far-infrared technology.

Materials Sciences Laboratory: Development of new materials; metal matrix composites and new forms of carbon; test and evaluation of $\mathrm{graphite}$ and ceramics in reentry; spacecraft materials and electronic components in nuclear weapons environment; application of fracture mechanics to stress corrosion and fatigue-induced fractures in structural metals.

Space Sciences Laboratory: Atmospheric and ionospheric physics, radiation from the atmosphere, density and composition of the atmosphere, aurorae and airglow; magnetospheric physics, cosmic rays, generation and propagation of plasma waves in the magnetosphere; solar physics, studies of solar magnetic fields; space astronomy, x-ray astronomy; the effects of nuclear explosions, magnetic storms, and solar activity on the earth's atmosphere, ionosphere, and magnetosphere; the effects of optical, electromagnetic, and particulate radiations in space on space systems. 
Aerospace Report No. ATR-79(7824)-2

ARGON-ION CONTAMINATION OF THE PLASMASPHERE

Prepared by

Y. T. Chiu, J. M. Cornwall (Consultant), J. G. Luhmann, and Michael Schulz

Space Sciences Laborato ry

15 July 1979

LABORATORY OPERATIONS

THE AEROSPACE CORPORATION

El Segundo, Calif. 90245

Prepared for

ARGONNE NATIONAL LABORATORIES

Argonne, Illinois 60439

Contract No. 31-109-38-5075

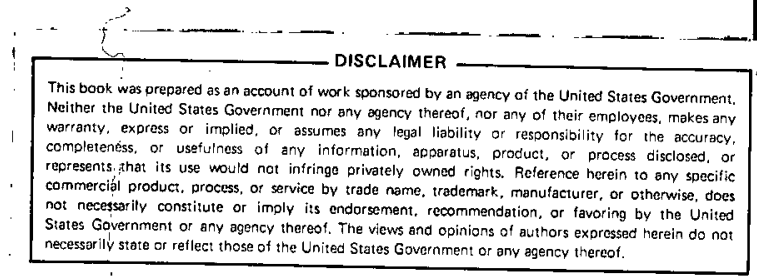




\section{THIS PAGE \\ WAS INTENTIONALLY \\ LEFT BLANK}


Report No.

ATR-79(7824)-2

ARGON-ION CONTAMINATION

OF THE PLASMASPHERE

Prepared

Y.T Chin

Y. T. Chin

D. Luthern

J. G. Luhmann

Approved

Fred Morse

F. A. Morse, Head

Atmospheric Sciences Dept.
J. M. Cornwall

J. M. Cornwall

Michael Shul
G. A. Paulikas, Director

Space Sciences Laboratory

iii 
THIS PAGE

WAS INTENTIONALLY

LEFT BLANK 


\section{ABSTRACT}

This paper applies present observational and analytic knowledge on effects of plasma beam interaction with the magnetosphere to the plasmasphere contamination problem of the argon ion engine exhaust expected to be deposited in the magnetosphere during the construction phase of the Satellite Power System. Effects of plasmasphere, ionosphere, and radiation belt modifications are discussed. 
THIS PAGE

\section{WAS INTENTIONALLY \\ LEFT BLANK}


CONTENTS

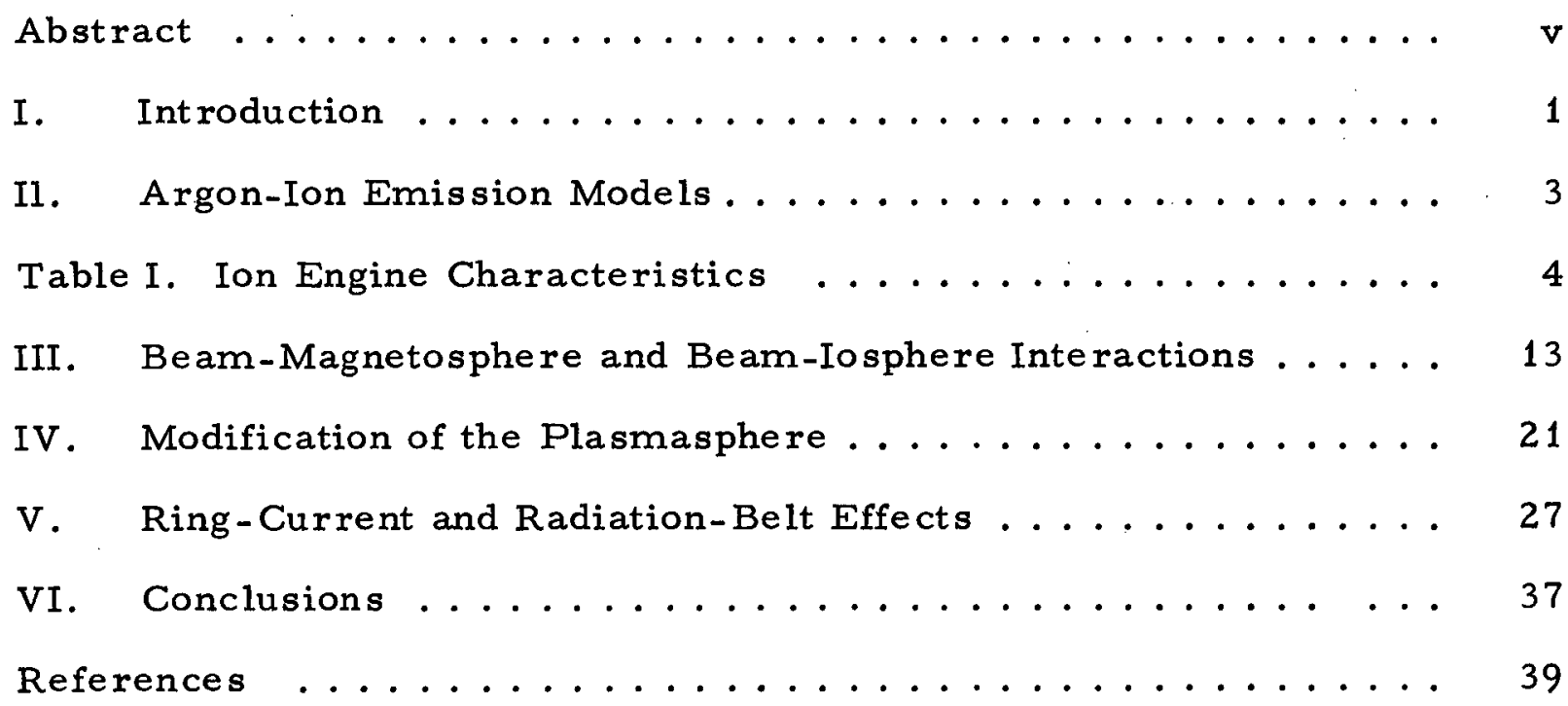


THIS PAGE

WAS INTENTIONALLY

LEFT BLANK 


\section{FIGURES}

1. A schematic representation of the cargo orbit transfer vehicle (COTV) argon-ion emission scenario at $L=4 \ldots \ldots \ldots$

2. Argon propellant mass necessary for the transport of different payload masses between $350 \mathrm{~km}$ altitude and

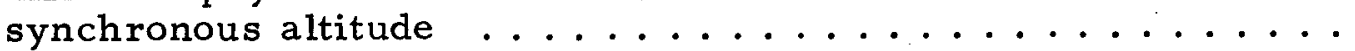

3. Fraction of mission lifetime $\mu$ spent at various geocentric

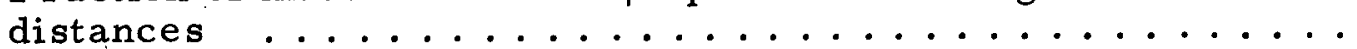

4. Number of $\mathrm{Ar}^{+}$ions released at various geocentric distances for payload mass of $10 \mathrm{~kg}$, compared to the number of ambient electrons in a dipole field shell two

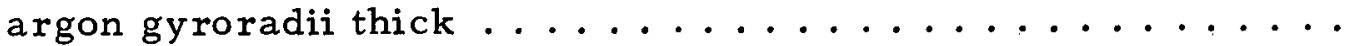

5. Comparison of charge exchange and coulomb lifetimes of $\mathrm{Ar}$ ions at various geocentric distances in the equatorial

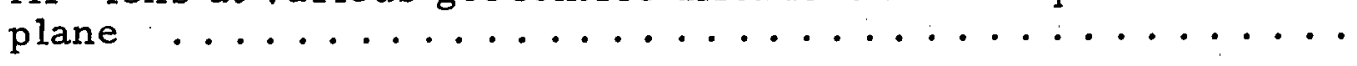

6. : Schematic representation of the beam-magnetosphere and beam-ionosphere interactions involve propagation and reflection of an Alfven wave ................

7. Density distribution along the $L=4$ dipole field line for the natural plasmasphere model of Chiu et al. (1979b) .......

8. Calculated $\mathrm{L}=4$ ion density distributions for various $\mathrm{Ar}^{+}$ concentrations at the $500 \mathrm{~km}$ altitude boundary and several thermal models

9. Lowest-order estimates for the normalized growth rate as a function of normalized wave frequency, for various relative concentrations $\left(\mathrm{N}_{\mathrm{Ar}} / \mathrm{N}_{\mathrm{H}}\right)$ of argon and hydrogen plasma 


\section{Introduction}

As is pointed out in the review of environmental effects of space systems (paper II A.), transportation of large payloads, such as that of the proposed satellite power system, from low earth orbit to higher space orbit; e.g., geosynchronous orbit, by ion engines implies substantial modifications of the magnetospheric environment. This is because the mass of exhaust plasma approaches or exceeds that of the local natural magnetospheric plasma. In this paper, we shall examine the basic physical scenarios of magnetospheric modification by this artificially injected plasma. The emphasis will be placed on identifying the physical mechanisms of the modifications rather than on a specific enumeration of space-based and earth-based systems which may be affected by these modifications. A discussion of the effects on systems operations can be found in Chiu et al. (1979a).

Despite the title of this paper, the effects that we shall deal with are sufficiently general that they are neither limited to argon ions nor to plasma exhaust products of ion engines alone. The latter is true because of the critical velocity phenomenon (Alfvén and Arrhenius, 1976; Angerth et al., 1962; Danielsson and Brenning, 1975; Möbius et al., 1979) which suggests that a neutral particle cloud moving with velocity relative to the ambient plasma greater than $v_{c} \equiv\left(|e| v_{i} / m\right)^{1 / 2}$ is apt to be ionized, where $|e| V_{i}$ is the ionization potential energy of the neutral particles of mass $m$. By noting the masses ( 10-50 atomic numbers) and the ionization potentials $(\sim 1-10 \mathrm{eV})$ of the usual chemical rocket propellant elements, the reader can easily verify that their critical velocities $v_{c}$ are $\sim 10 \mathrm{~km} / \mathrm{sec}$, which 
are frequently exceeded for cases of rocket propellants in the magnetosphere where spacecraft velocities are already $\sim 10 \mathrm{~km} / \mathrm{sec}$ relative to the background magnetospheric plasma. Therefore, if the critical velocity phenomenon, observed in the laboratory, is applicable in space, the operation of chemical rockets in the magnetosphere will generate ion clouds which behave in much the same manner as the argon-ion clouds to be dealt with in this paper. In this regard, the magnetospheric modifications considered here are applicable to large-scale injections of high-speed $\left(>v_{c}\right)$ rocket exhaust in the magnetosphere, irrespective of whether the booster engines are ion engines or not.

The subject of magnetospheric modification by a massive plasma cloud is obviously very complicated and, to a major extent, unsolved. An attempt to trace the dynamical history of the artificial plasma is too difficult a task here, although it would eventually have to be faced. Our efforts here will be limited to the initial phases of the problem for which we ignore the question of the long-term fate of the artificially injected material.

Section II of this paper will present a simplified emission scena rio of argon-ion engines for the proposed satellite power system which will be used as the standard for comparison. Sections III, IV and V will address details of modifications due to interactions of the plasma beam with the magnetosphere, modifications of the plasmaspheric composition and density, and the modification of radiation belt dosage, respectively. 


\section{Argon-Ion Emission Models}

The current technology of ion engines is still evolving (Kauffman, 1974; Byers and Rawlin, 1976); therefore, the parameters of argon-ion engine operations in space must largely be regarded as uncertain at present, although it is by now fairly firm that the most economical and environmentally safe propellant is argon. According to the reference system report of the satellite power system concept development and evaluation program (DOE/ER-0023, U.S. Department of Energy and National Aeronautics and Space Administration, January 1979), a rgon-ion engines of specific impulse $13000 \mathrm{sec}$. are projected to perform major propulsion and stationkeeping duties for cargo orbit transfer vehicles as well as for the spacecraft at geosynchronous orbit. Other considerations (Byers and Rawlin, 1976), with perhaps less stringent requirements on projected advances on the technology of ion engines, assumed 5000 sec. specific impulse as standard for comparison. Some projected characteristics of these two options of ion engine operation are listed in Table $I$. Since the reader may easily scale the results of this paper by means of Table I, we shall, henceforth, consider option 2 only.

From Table I, it is seen that the ion beam exhaust is a very dense, but fairly cool plasma whose streaming kinetic energy $(0.5 \mathrm{keV})$ far exceeds the thermal energy. Further, in order to propel the cargo orbittransfer vehicles (COTV) to geosynchronous orbit, the argon plasma beam will be directed perpendicular to the geomagnetic field at the equatorial plane in the azimuthal direction (Figure 1). Plasma beams propagating perpendicular to the geomagnetic field entails very interesting dynamical interactions with the magnetosphere-ionosphere system. This topic will be treated in the next section. 
Table I. Ion Engine Characteristics

Option 1

Specific impulse (sec)

$\mathrm{Ar}^{+}$kinetic energy $\left(\mathrm{keV} / \mathrm{Ar}^{+}\right)$

Art streaming speed $(\mathrm{km} / \mathrm{sec})$

Current density $\left(\mathrm{amp} / \mathrm{cm}^{2}\right)$

Temperature $\left({ }^{\circ} \mathrm{K}\right)$

Beam density $\left(\mathrm{cm}^{-3}\right)$

Beam diameter at exit $(\mathrm{cm})$

Beam spread at exit (deg.)

Number of engines required for SPS/COTV
13000

3.5

130

2. $5 \times 10^{-2}$

$\sim 1000$

$\sim 1.5 \times 10^{10}$

100

$\sim 10^{\circ}$

$\sim 300$
Option 2

5000

0.5

50

2. $5 \times 10^{-2}$

$\sim 1000$

$\sim 4 \times 10^{10}$

100

$\sim 10^{\circ}$

$\sim 800$ 


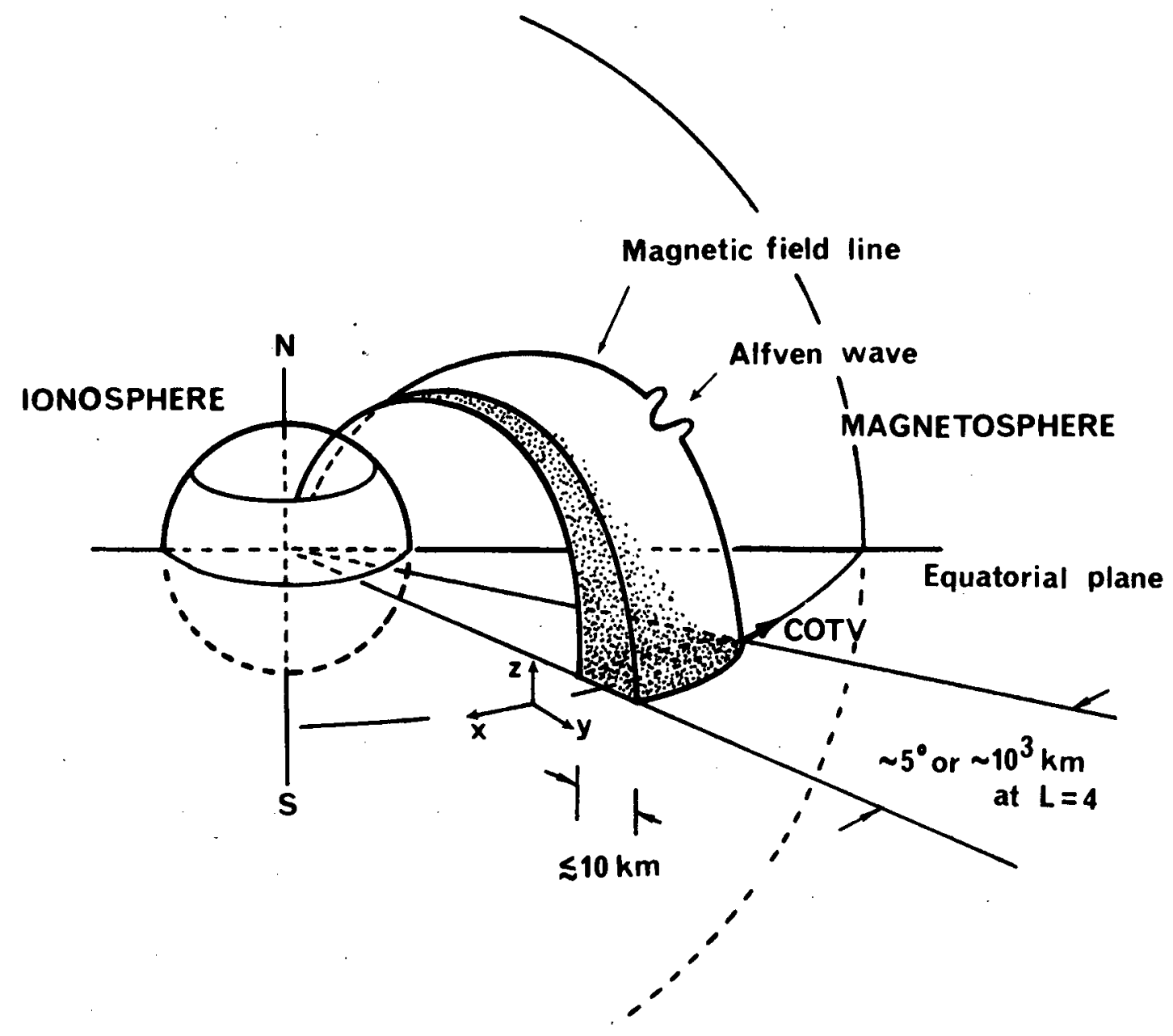

Figure 1. A schematic representation of the cargo orbit transfer vehicle (CO'TV) argon-ion emission scenario at $L=4$. The coordinate system used in this paper is also defined. The ion beam trailing the COTV is roughly $1000 \mathrm{~km}$ long and $\sim 10 \mathrm{~km}$ in width. 
For consideration of emission parameters, Figure 2 (taken from Chiu et al., 1979a) shows the relationship between payload mass and argon propellant mass needed to transport the payload from low earth orbit (350 $\mathrm{km}$ altitude) to synchronous altitude with an accompanying orbital plane change of $28.5^{\circ}$. Obviously, the amount of propellant required for a given payload depends on the ion-beam streaming speed. For satellite power system payload of $\sim 10^{7} \mathrm{~kg}$, it will be necessary to expend $\sim 10^{6} \mathrm{~kg}$ of argon propellants for option 2 in Table I. This is $\sim 1.5 \times 10^{31} \mathrm{Ar}^{+}$ions, roughly comparable to the total content of the natural plasmasphere and ionosphere above $500 \mathrm{~km}$. The exhaust deposition rate in terms of the fraction $\mu$ of mission lifetime, which is nominally $\sim 130$ days, is shown as a function of geocentric radius $R$ on Figure 3 (Chiu et al., 1979a). Thus, $80 \%$ of the total própellant content is released in the plasmasphere, $\mathrm{R} \leq 4 \mathrm{R}_{\mathrm{E}}$. The number of $\mathrm{Ar}^{+}$released at a given geocentric distance for a payload mass of $10^{7} \mathrm{~kg}$ is shown on Figure 4; for comparison, the number of ambient electrons lying within a flux shell of thickness equal to twice the argon gyroradius at a given distance $R$ is also shown. The energy content released into a given shell dominates the ambient energy. content, however, since the streaming $\mathrm{Ar}^{+}$kinetic energy is considerably greater than the ambient thermal energy.

The fate of the injected $\mathrm{Ar}^{+}$depends largely on the plasma dyramics of the $\mathrm{Ar}^{+}$cloud interacting with the ambient magnetosphere. Some features of this dynamical interaction will be addressed in the next section. For the majority of the injected $\mathrm{Ar}^{+}$that are trapped in the plasmasphere, their energy $\left(\sim 500 \mathrm{eV}\right.$ per $\left.\mathrm{Ar}^{+}\right)$degrades via at least two routes. First, the propagation of the $\mathrm{Ar}^{+}$plasma across the geomagnetic field drives 


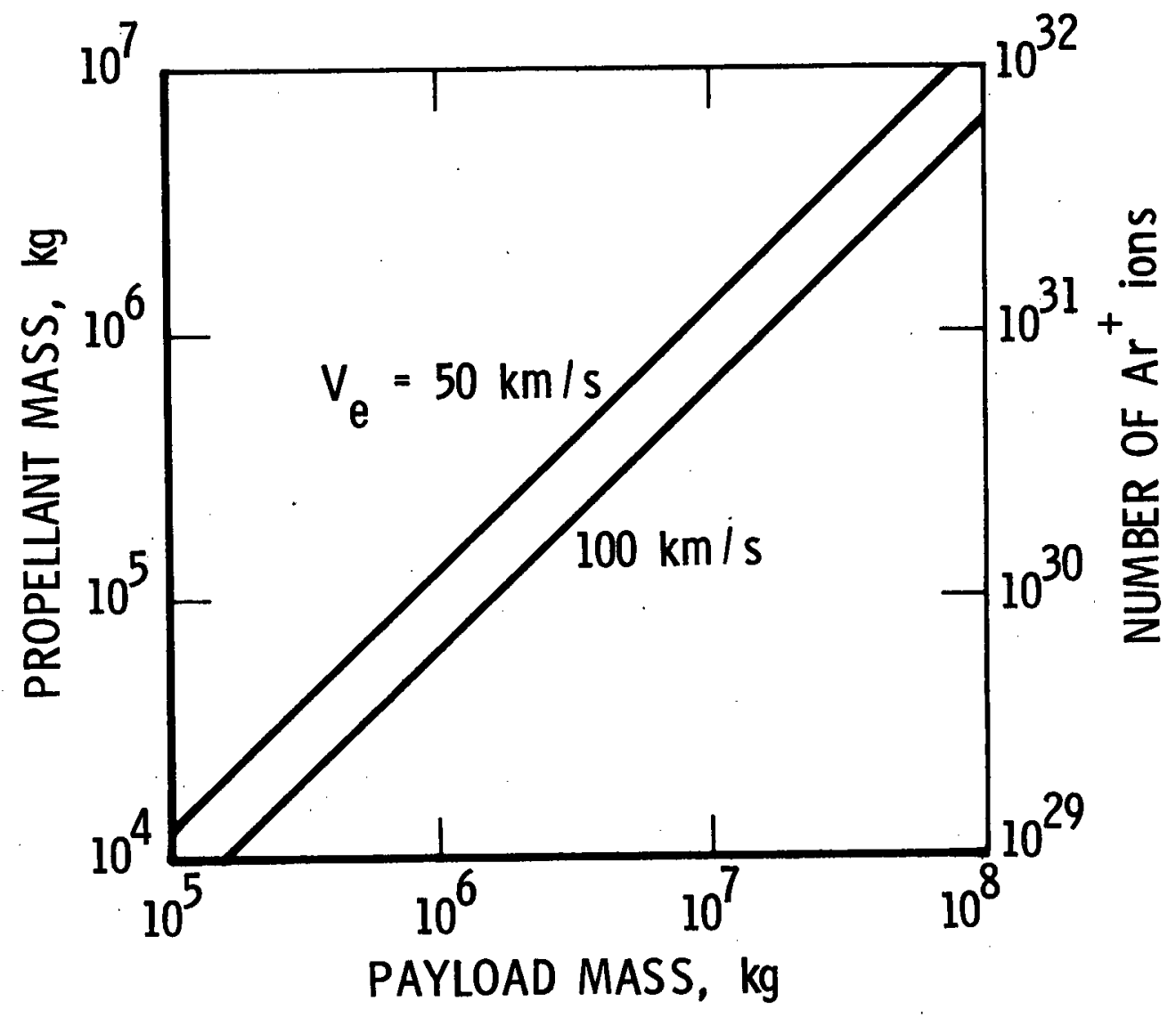

Figure 2. Argon propellant mass necessary for the transport of different payload masses between $350 \mathrm{~km}$ altitude and synchronous altitude. The orbital inclination is as sumed to change from $0^{\circ}$ to $28.5^{\circ}$ during transport. 


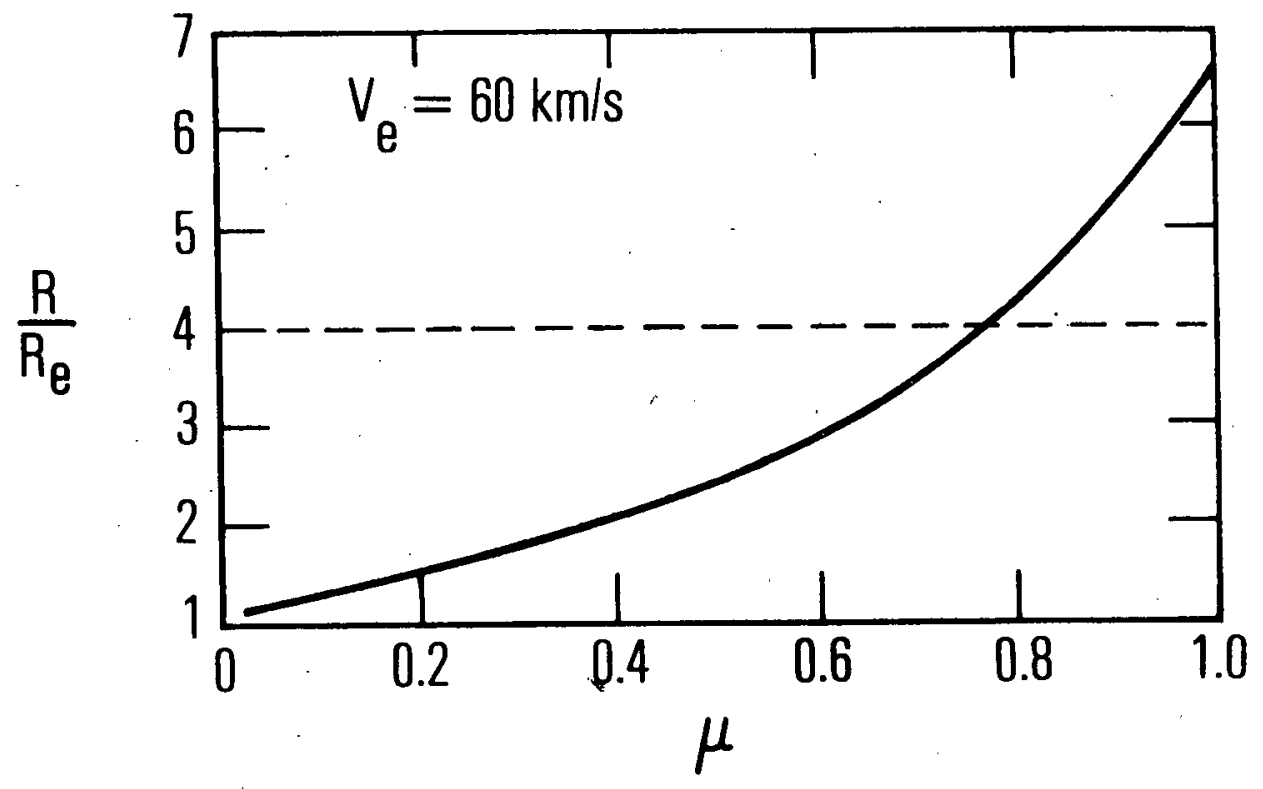

Figure 3. Fraction of mission lifetime $\mu$ spent at various geocentric distances. 


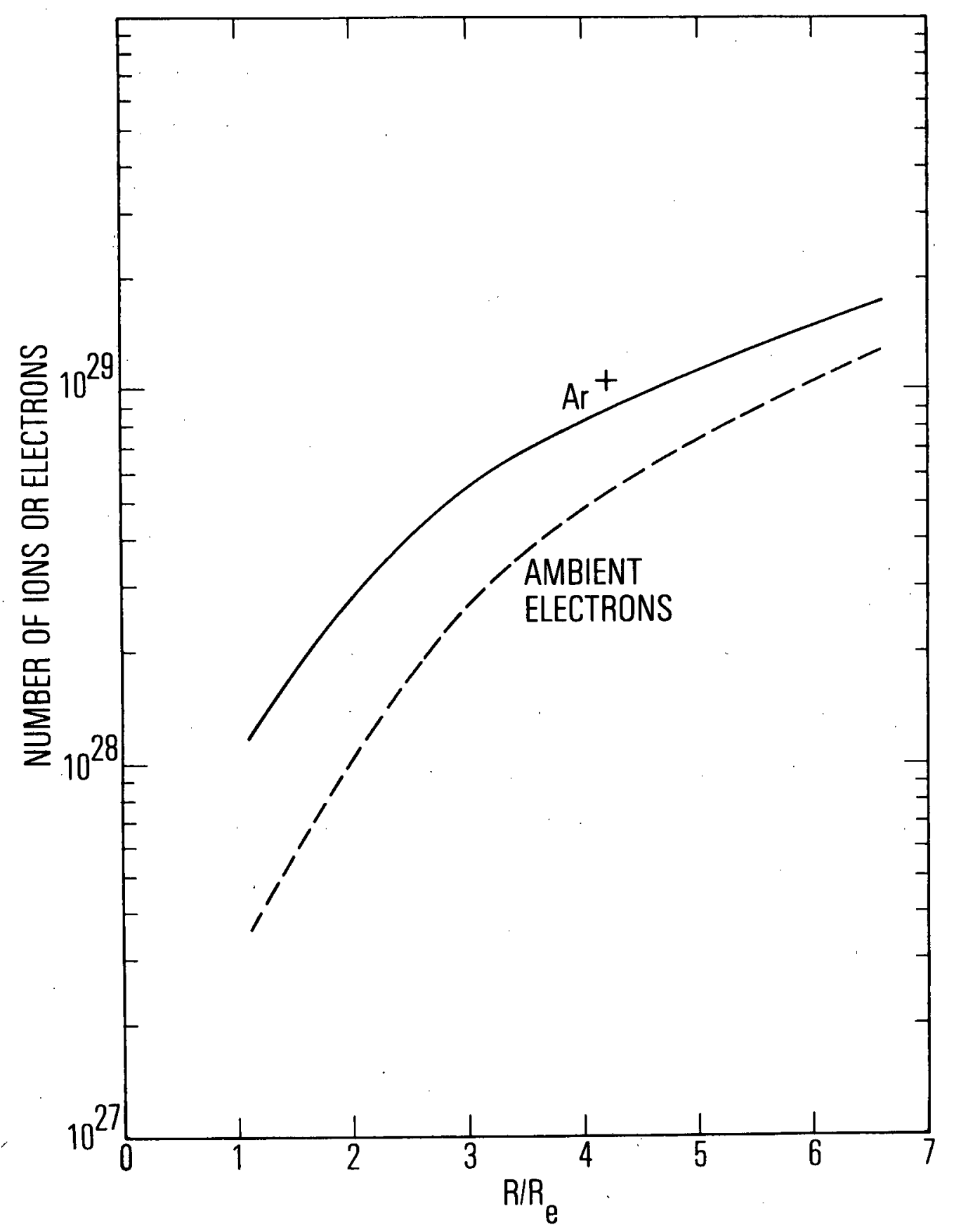

Figure 4. Number of $\mathrm{Ar}^{+}$ions released at various geocentric distances for a payload mass of $10^{7} \mathrm{~kg}$, compared to the number of ambient electrons in a dipole field shell two argon gyroradii thick.

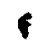


ionospheric currents which dissipate a substantial fraction of the $\mathrm{Ar}^{+}$ streaming energy (see next section). Second, long range Coulomb collisions with ambient electrons degrade the kinetic energy into ambient thermal energy without causing substantial changes in pitch-angle because these Coulomb collisions are primarily forward scatterings. For the energetic $\mathrm{Ar}^{+}$to be physically lost from the magnetosphere, they will have to suffer charge exchange collisions which would allow the neutral argon atom to escape the plasmasphere. Figure 5 shows the comparison of charge exchange lifetimes of $\mathrm{Ar}^{+}$in the plasmasphere $(L \geq 2)$ with the thermalization lifetime due to Coulomb collisions. Note that the charge exchange lifetimes $(\sim 100 \mathrm{hrs})$ are comparable to the average duration between magnetospheric substorms which typically defines the lifetimes of the natural magnetospheric plasma. Thus, we expect that the injected Ar plasma will substantially modify the magnetosphere with a time constant roughly equal to several days until a new magnetospheric substorm sweeps out the accumuition, if the substorm mechanism remains effecive under the modified circumstances. 


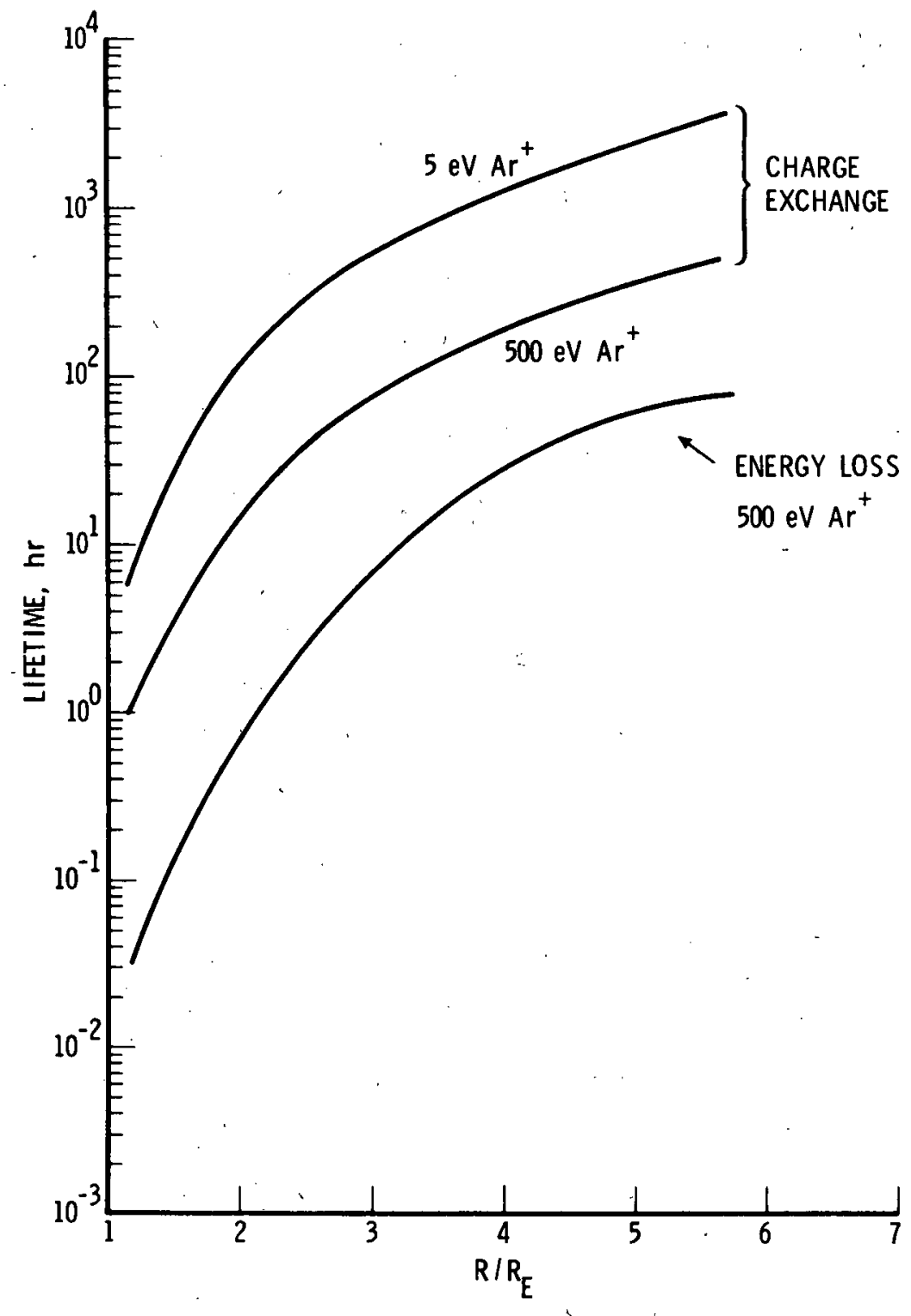

Figure 5. Comparison of charge exchange and coulomb lifetimes of $\mathrm{Ar}^{+}$ions at various geocentric distances in the equatorial plane. The charge exchange cross section used in the calculation of the lifetime was measured by Gilbody and Hasted (1956). The plasmasphere is as sumed to occur at. $4 \mathrm{Re}$. 
III. Beam-Magnetosphere and Beam-Ionosphere Interactions

The physics of a plasma beam propagating transverse to a homogeneous vacuum magnetic field is very simple: if the beam is sufficiently dense so that polarization currents can maintain the charge separation electric field necessary to satisfy $\vec{E}+\frac{1}{C} \vec{v} \times \vec{B}=0 \quad(\vec{v}$ is the beam velocity), the beam will propagate across the magnetic field. An alternative view of the effects of the polarization electric field $\vec{E}$ seen by a co-moving observer above is that, in the coordinate system of the stationary magnetic field outside of the cloud, the plasma cloud, under the force of $\vec{E}$, appears to be drifting with a velocity $\vec{v}=c(\vec{E} \times \vec{B}) / B^{2}$. But $\vec{v}$ above is also the drift velocity of magnetic field lines in the cloud induced by the electric field $\vec{E}$; hence, the field lines in the cloud are said to be "frozen" into the plasma, drifting with velocity $\vec{v}$ relative to the field lines outside of the cloud. For this condition to apply, the beam density $\mathrm{n}_{\mathrm{A}}$ must satisfy

$$
4 \pi \mathrm{n}_{\mathrm{A}} \mathrm{m}_{\mathrm{A}} \mathrm{c}^{2} / \mathrm{B}^{2} \gg 1
$$

where $\mathrm{m}_{\mathrm{A}}$ is the argon-ion mass (Curtis and Grebowsky, 1979). Numerically, (1) yields (500-30000) $\mathrm{n}_{\mathrm{A}}\left(\mathrm{cm}^{-3}\right) \gg 1$ for $2 \leq \mathrm{L} \leq 4$, which would seem to be well-satisfied for the beam parameters of Table I; if so, the beam simply moves out of the magnetosphere to be dissipated in space. Unfortunately, this conclusion is completely false. The magnetosphere, and particularly the ionosphere, cannot be considered in terms of a vacuum magnetic field. Plasmas in the magnetosphere, and especially 
in the ionosphere, act to short out the charge-separation electric field $\vec{E}$ and stop the beam in a distance of the order of $1000 \mathrm{~km}$. As a consequence, the beam dissipates its energy in a rather localized region and may well

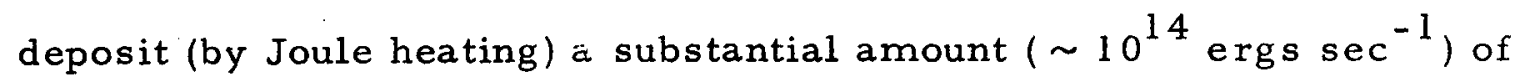
energy. in a relatively small area of the ionosphere connected by magnetic field line to the argon beam (see Figure 1), long before the majority of Ar ${ }^{+}$ ions are physically present in the ionosphere. Later in this section, we give some details of this picture.

Granted that the argon beam is stopped in the magnetosphere, it begins to act like a man-made ring current of $\leqslant \mathrm{keV}$ ions, with a residual pitch-angle anisotropy left over from the initial injection nearly perpendicular to $\vec{B}$. (Just what energy and anisotropy are left after the beamplasma interactions described in this section is not known at present; they need to be evaluated in order to give a more precise picture of their influences on systems such as increase in airglow and changes in radiation belt dosage levels.) This ring current acts much like a natural one, subject to charge exchange, Coulomb scattering (Section II), and wave-particle interactions (see below). If charge exchange is the dominant loss process (Coulomb scattering or other forms of energy loss does not remove argon), this ring current may have $\sim 10^{30}$ ions in it, comparable to the natural ring current. A major difference is that the argon ring current is mostly inside the plasmasphere, while the natural ring current penetrates perhaps one earth radius inside the plasmasphere with the rest outside. Nonetheless, there could be substantial overlap between the ring currents, especially during storm times when the plasmasphere is eroded and the natural ring current driven in by convection electric fields. The argon ring 
current can make a substantial contribution to the plasmaspheric pressure and hence to the currents which flow there (both across and along $\vec{B}$ ); this will significantly stiffen the plasmasphere to deformations associated with enhanced storm-time convection electric fields and diminish the strength of electrostatic radial diffusion. The result will be (othe $r$ things being unchanged) a buildup of ring-current particles and inhibition of inward transport of higher-energy particles.

Aside from the microscopic processes alluded to above, there a re many possible beam-plasma interactions. These involve not only the dense (1-100 $\left.\mathrm{cm}^{-3}\right)$, relatively cold $(\lesssim 1 \mathrm{eV})$ the rmal plasma, but also ringcurrent and radiation-belt particles whose energies range from keV's to $\mathrm{MeV}^{\prime} \mathrm{s}$. A partial list of important processes (in order of increasing time scale): cross-field current-driven instabilities (time scale $R_{e} / v \sim$ milliseconds, where $R_{e}$ is the electron Larmor radius); ion electrostatic modes (time scale $=\omega_{A}^{-1} ; \omega_{A}$ is the $\mathrm{Ar}^{+}$plasma frequency $\approx 10^{-2} \mathrm{sec}^{-1}$ ); proton electromagnetic cyclotron $(E M C)$ modes (time scale $\approx \Omega_{\mathrm{p}}^{-1} \approx 0.1$ sec). These are important because they can cause local transfer of energy from the beam to the plasmasphere (first two modes), or because they affect natural magnetospheric processes. Thus the proton EMC mode is inhibited by heavy ions (Cornwall and Schulz, 1971). This may mean a buildup both of ring-current protons and of relativistic electrons, which otherwise would be precipitated into the ionosphere by the EMC instability (see Section V). There are also magnetohydrodynamic instabilities of considerably larger time scale (e.g., Perkins et al., 1973), which cause deformations of the beam (striations, etc.), but which probably do not 
transfer much energy from beam to plasmasphere. The free energy for these instabilities is not only the beam kinetic energy, but the energy associated with pitch-angle anisotropies and spatial gradients.

In the remainder of this Section we give a brief description of the physics of stopping the beam, and of dissipating some of its energy in the ionosphere. The basic physics is well-known, and was worked out by Scholer (1970) and Pilipp (1971) in connection with the HEOS-I release of an ionized barium cloud at $L=12$ (Haerendel and Lüst, 1970). This high-altitude release had, as will the argon engines, a high initial $\beta$ $\left(=8 \pi P_{\perp} / B^{2}\right.$, where $P_{\perp}$ is the pressure perpendicular to the field lines of the injected plasma). The beam expands rapidly, in a direction perpendicular to $\vec{v}$ and to $\vec{B}$, to the point where $\beta \lesssim 1$. Of course, the beam also spreads without constraint (except for mirroring forces) along $\vec{B}$.

One could calculate the final beam spread $\Delta y$ in the $y$-direction (or $\vec{v} \times \vec{B}$ direction; see Figure 1) using zero-Larmor-radius magnetohydrodynamics, that is, by equating $P_{\perp}$ (including thermal pressure $\mathrm{n}_{\mathrm{A}} \mathrm{kT}$ plus dynamic pressure $\frac{1}{2} \mathrm{n}_{\mathrm{A}} \mathrm{m}_{\mathrm{A}} \mathrm{v}^{2} \tan ^{2} \theta$ ) to the asymptotic plasmaspheric pressure, which is essentially $B^{2} / 8 \pi$. Our flux conservation requires $n_{A}=n_{0 A} A / \Delta z \Delta y$, where $n_{0 A}$ and $A$ are the initial beam density and area (see Table I) and $\Delta z$ is the beam spread along the field. Assuming $\Delta z z \Delta y$, one finds that $\Delta y$ is less than $\sim 1 \mathrm{~km}$ for $\mathrm{L}<4$, much smaller than the argon Larmor radius $R_{A}$. In effect, this calculation of the confinement of gyration centers tells us that $\Delta y$ is of the order of the argon Larmor radius $(40-80 \mathrm{~km}$ at $L=4)$ because the gyration centers are confined to a $\Delta y$ much smaller than the argon Larmor 
radius, at least for the first ten or so Larmor radii downstream from the nozzle; past this, the $\sim 10^{\circ}$ angular divergence of the beam could produce substantially larger $\Delta y$ (as long as (1) continues to hold).

In first approximation, then, we have a beam of $\Delta y \approx R_{A}$ propagating across the earth's field as shown in Figure 6. In this Figure, the dotted lines show schematically earth's magnetic field lines at various times. The condition $\vec{E}+\frac{1}{c} \vec{v} \times \vec{B}=0$ means that the se lines a re frozen into the plasma beam at the equator; their distortion is an Alfvén wave $(t=1$, in Figure 6). At $t=2$, the wave reaches the ionosphere, where the foot of the field line slips, because of the ionosphere's finite conductivity; the wave then reflects back to the beam $(t=3,4)$.

The field lines act somewhat like rubber bands, tending to retard the cloud. The physical mechanism is that the polarization charges responsible for $\vec{E}$ move along the field lines at the Alfvén speed $v_{A}$, accelerating magnetospheric plasma and transferring momentum out of the beam. Ultimately, the Alfvén wave reaches the ionosphere and drives dissipative Pedersen currents (in the absence of dissipation, the argon beam would oscillate like a mass on a rubber band field line).

Let $M_{A}$ be the mass density of the argon beam, integrated along field lines passing through the beam:

$$
\mathrm{M}_{\mathrm{A}}=\int \mathrm{dz} \mathrm{n}_{\mathrm{A}} \mathbf{m}_{\mathrm{A}}
$$

When this mass density is equal to the mass per unit area incorporated by the Alfvén wave, namely $2 \mathrm{v}_{\mathrm{A}} \tau \mathrm{n}_{0} \mathrm{~m}_{\mathrm{p}}$, the beam is essentially stopped. 


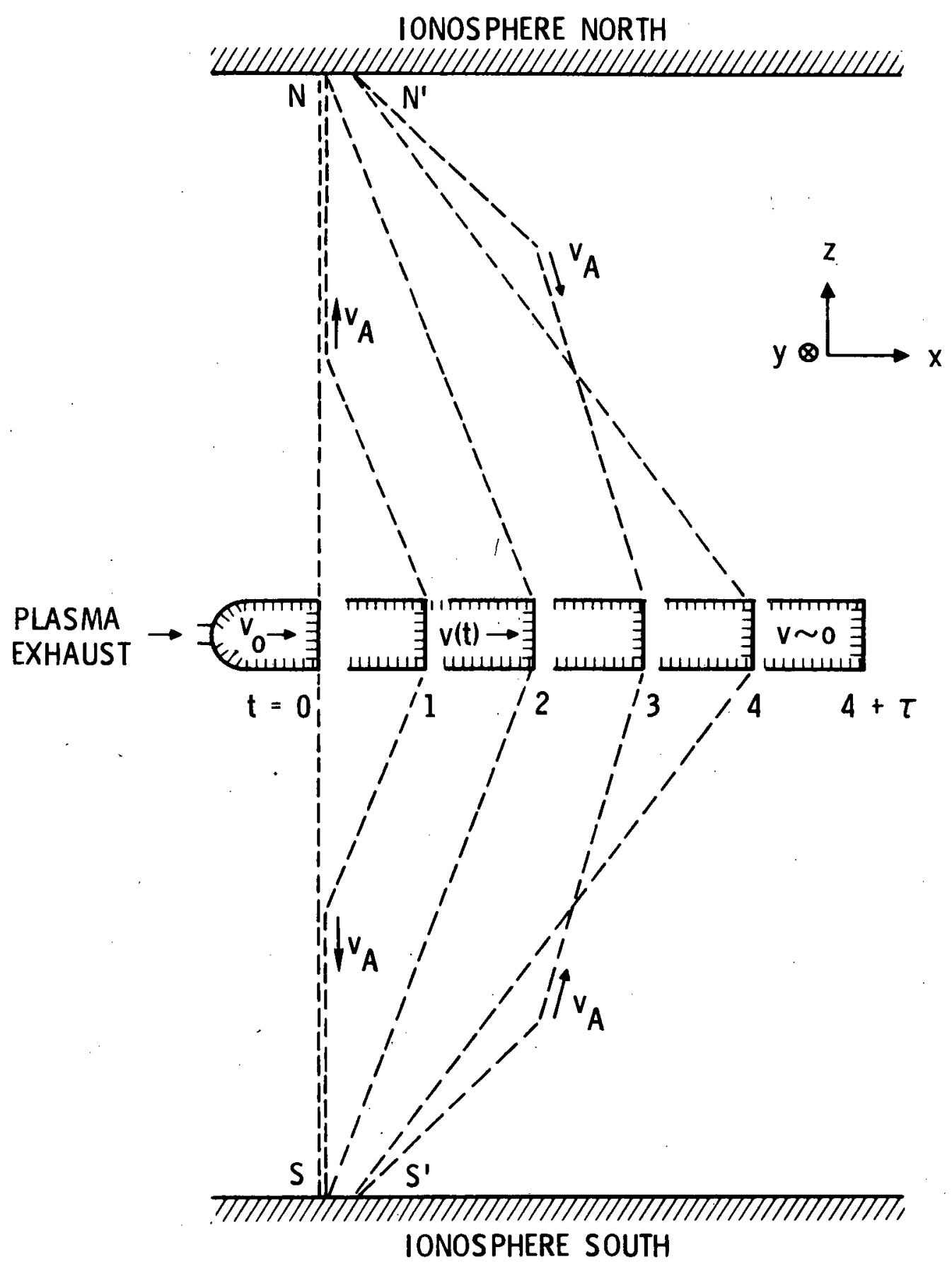

Figure 6. Schematic representation of the beam-magnetosphere and beam-ionosphere interactions involve propagation and reflection of an Alfven wave. The curvature of the magnetic field lines and the gradient of magnetic field intensity are not represented in this model. 
Here $\tau$ is the time it takes the Alfvén wave to travel a distance $v_{A} T$, and $\mathrm{n}_{0} \mathrm{~m}_{\mathrm{p}}$ is the magnetospheric mass density per unit volume. For the argon beam, $\mathrm{M}_{\mathrm{A}} \approx 5 \times 10^{-13} \mathrm{gm} \mathrm{cm}^{-2}$ at $\mathrm{L}=4 ;$ if $\mathrm{v}_{\mathrm{A}} \approx 3 \times 10^{7} \mathrm{~cm}$ $\sec ^{-1}$ and $\mathrm{n}_{0} \approx 10^{3} \mathrm{~cm}^{-3}$, this gives $T \approx$ few seconds. The beam's velocity behaves like $v \simeq v_{0} e^{-t / T}$, so the beam can only travel a distance of the order of $v_{0} \tau \leqslant 10^{3} \mathrm{~km}$. In this example, the beam momentum is soaked up by magnetospheric plasma extending at most a few thousand kilometers down the field line on either side of the beam.

The ionosphere "feels" the beam after a time $\approx \ell / \mathrm{v}_{\mathrm{A}} \approx$ tens of seconds ( $\ell$ is the length of the field line). The electric field $E_{I}$ imposed on the ionosphere differs from the $\frac{1}{C} \vec{v} \times \vec{B}$ field, mapped to the ionosphere, for two reasons: first, the electric field diminishes along the field line by a factor of order $e^{-\ell / v_{A} \tau}$, and second, the electric field is partly reflected at the ionosphere (to make the upgoing Alfvén wave at $t=3,4$ in Figure 6 ). Scholer gives the relation

$$
\begin{gathered}
E_{I}=[2 /(1+\chi)] E_{\text {out }} \\
X=4 \pi \Sigma_{p} v_{A} / c^{2}
\end{gathered}
$$

where $E_{\text {out }}$ is the field just outside the ionosphere, $E_{I}$ the field in the ionosphere, and $\Sigma_{\mathrm{p}}$ the ionospheric Pedersen conductivity. It is somewhat hazardous to make numerical estimates with exponential factors floating a round, but it would be surprising if the ionospheric time scale for dissipation, ${ }^{T} \mathrm{I}$, defined by 


$$
\tau_{I}=M_{A} \cdot v_{0}^{2} / 2 \Sigma_{p} E_{I}^{2}
$$

were very much greater than $\tau$. Taking the two time scales equal, the rate at which beam energy is dissipated in the ionosphere is roughly $\mathrm{M}_{\mathrm{A}} \mathrm{v}_{0}^{2} / 2 \tau \approx$ few ergs $/ \mathrm{cm}^{2} \mathrm{sec}$, and this might occur over an a rea of $10^{3}-10^{4} \mathrm{~km}^{2}$ in the ionosphere. If these numbers are roughly right, a substantial fraction of the beam's energy is dissipated in the ionosphere; otherwise, the beam energy goes into formation of magnetospheric waves and particle energization. In any case, we argue that the whole beam power of $\approx 5 \times 10^{15} \mathrm{ergs} / \mathrm{sec}$ will be dissipated in the magnetosphere and ionosphere. Indeed, observations of barium releases in the far magnetosphere seem to qualitatively confirm the above considerations (Scholer, private communications).

By comparison, a natural geomagnetic storm dissipates energy at a rate $\sim 10^{17} \mathrm{ergs} / \mathrm{sec}$, but only sporadically and over a much larger area. The dissipation rate per unit area for a storm is also a few ergs/ $\mathrm{cm}^{2}$ sec, comparable to the argon beam dissipation. The difference is that the argon beam will be present, day and night, for several months, while storms occur only every few days. It is, in fact, very difficult to find any hard information on the amount of naturally-occurring energy precipitated into the ionosphere over a span of time like four months (the ion-engine operational period), but it is not likely to exceed a few times $10^{22}$ ergs per month in moderately quiet times; this is of the order of magnitude of the energy released into the plasmasphere by the SPS ion engines. 


\section{Modification of the Plasmasphere}

In the previous sections it was found that the argon ions that are emitted by the ion engines will merge with the magnetospheric plasma rather than escaping through the magnetopause. Here the effect of the addition of these heavy ions and the accompanying heat input to the inner magnetosphere is considered. As a first approximation it is assumed that the ionized argon can be treated as a constituent of the steady-state plasmasphere with the extreme limits of the ionospheric concentration equal to the densities of hydrogen ions and oxygen ions at $500 \mathrm{~km}$ altitude. An earlier analytical model of the equilibrium plasmasphere derived by Chiu et al. $(1979 \mathrm{~b})$ is used to predict the changes that will occur due to the presence of argon ions and to the heating of the ambient plasma by the thermalization of the argon ions.

Chiu et al. (1979b) found that the observed plasmaspheric density between an altitude of $500 \mathrm{~km}$ and the dipole field line $\mathrm{L}=5$ can be adequately described by the expression

$$
\begin{aligned}
& n_{j}(s)=n_{j}(l)\left[B(s) T_{j}(l) / B(\ell) T_{j}(s)\right] \cdot \exp \int_{\ell}^{s} d s^{\prime} x \\
& {\left[q_{j}|e| E-m_{j}\left(G M_{E} / r^{2}+\Omega_{E}^{2} r\right) \hat{r}^{\prime} \cdot \hat{s}^{\prime}\right] / k T_{j}\left(s^{\prime}\right)}
\end{aligned}
$$

where $n_{j}$ is the density of ion species $j, s$ is the distance along the dipole field line measured from the equator $(s=0)$ to the ionosphere $(s=l), B$ is the magnetic field strength, $q|e|$ is the charge, $E$ is the electric field strength, $m$ is the ion mass, $G$ is the gravitational constant, $M_{E}$ is 
the mass of the earth, $\Omega_{E}$ is the angular rotation frequency of the earth, $\mathbf{k}$ is Boltzmann's constant, and $\mathbf{r}$ is the geocentric radial coordinate. The empirically determined temperature profile along a field line is given by

$$
T(s)=T_{0}+T_{1}\left(\frac{L-1}{L_{0}}\right)^{\alpha}\left(\frac{\ell-s}{\ell}\right)^{\beta}
$$

where $T_{0}, T_{1}, L_{0}, \alpha$ and $\beta$ are constants with the following nominal values: $\mathrm{T}_{0}=2000^{\circ} \mathrm{K}, \mathrm{T}_{1}=3000^{\circ} \mathrm{K}, \mathrm{L}_{0}=3, \alpha=1 / 2, \beta=1 / 2$. These authors considered a charge-neutral four constituent model composed of electrons, $\mathrm{H}^{+}, \mathrm{O}^{+}$and $\mathrm{He}^{+}$. Their result for $\mathrm{L}=4$ and the above temperature parameters is shown in Figure 7.

For the purpose of the present investigation, the minor constituent $\mathrm{He}^{+}$was omitted and argon ions were substituted as the fourth plasmasphere constituent in the model. As mentioned above, the ionospheric $(500 \mathrm{~km})$ concentration of argon ions was set equal to either the local concentration of hydrogen ions or of oxygen ions as nominal lower and upper limits. It must be recognized that these limits are at present arbitrary; eventually, these limits are set by a detailed study of the fate of $\mathrm{Ar}^{+}$in the magnetosphere. Several different fractional temperature increases were assumed. The results of the calculations for $L=4$ are shown in Figure 8 for comparison with Figure 7. It is seen from the left-hand panel of Figure 8 that merely the addition of the argon ions will not upset the normal distribution of the plasmaspheric hydrogen and oxygen; however, as shown by the righthand panel, the addition of heat by the argon-ion interaction with the ambient 


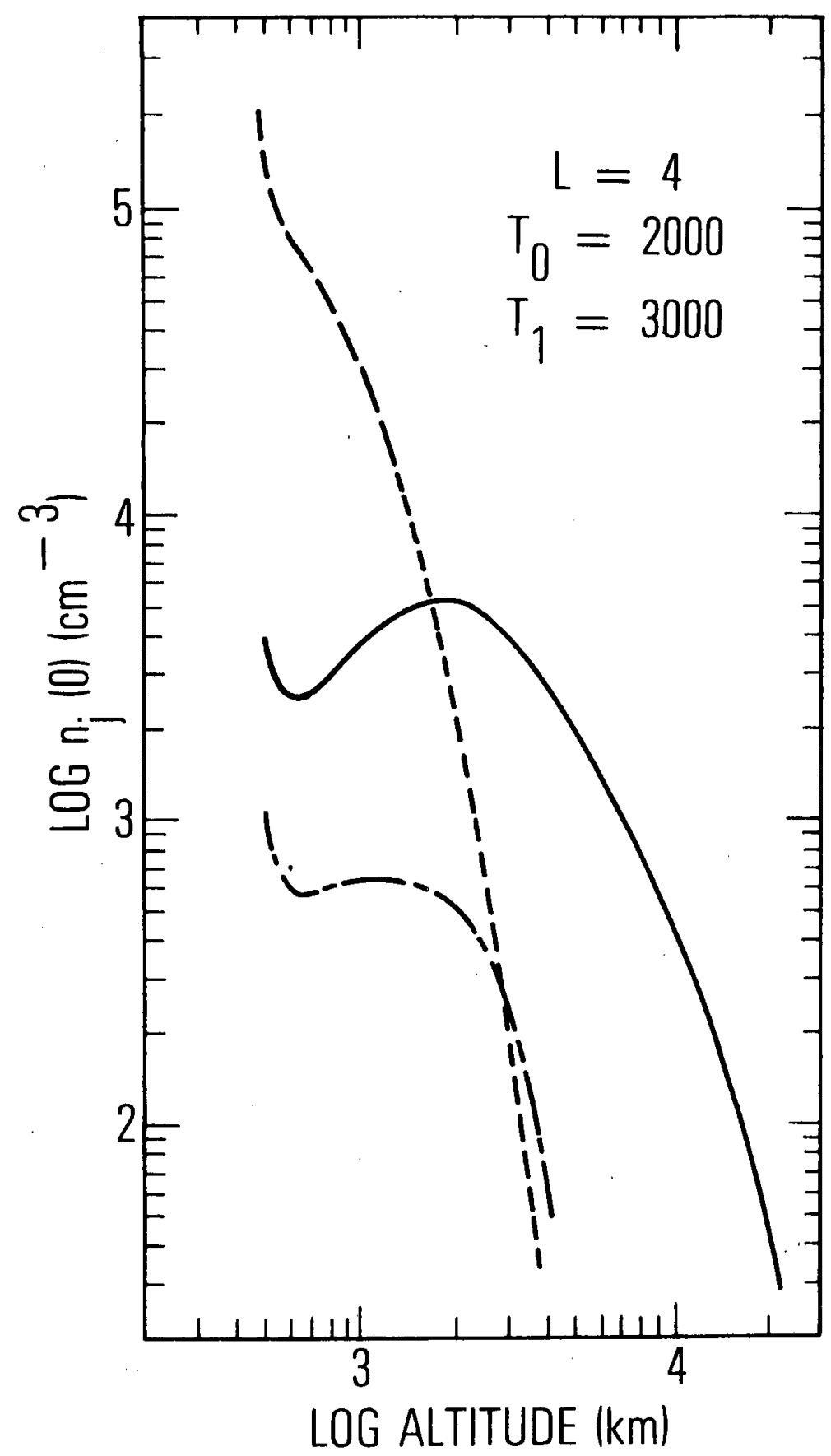

Figure 7. Density distribution along the $L=4$ dipole field line for the natural plasmasphere model of Chiu et al. (1979b). 


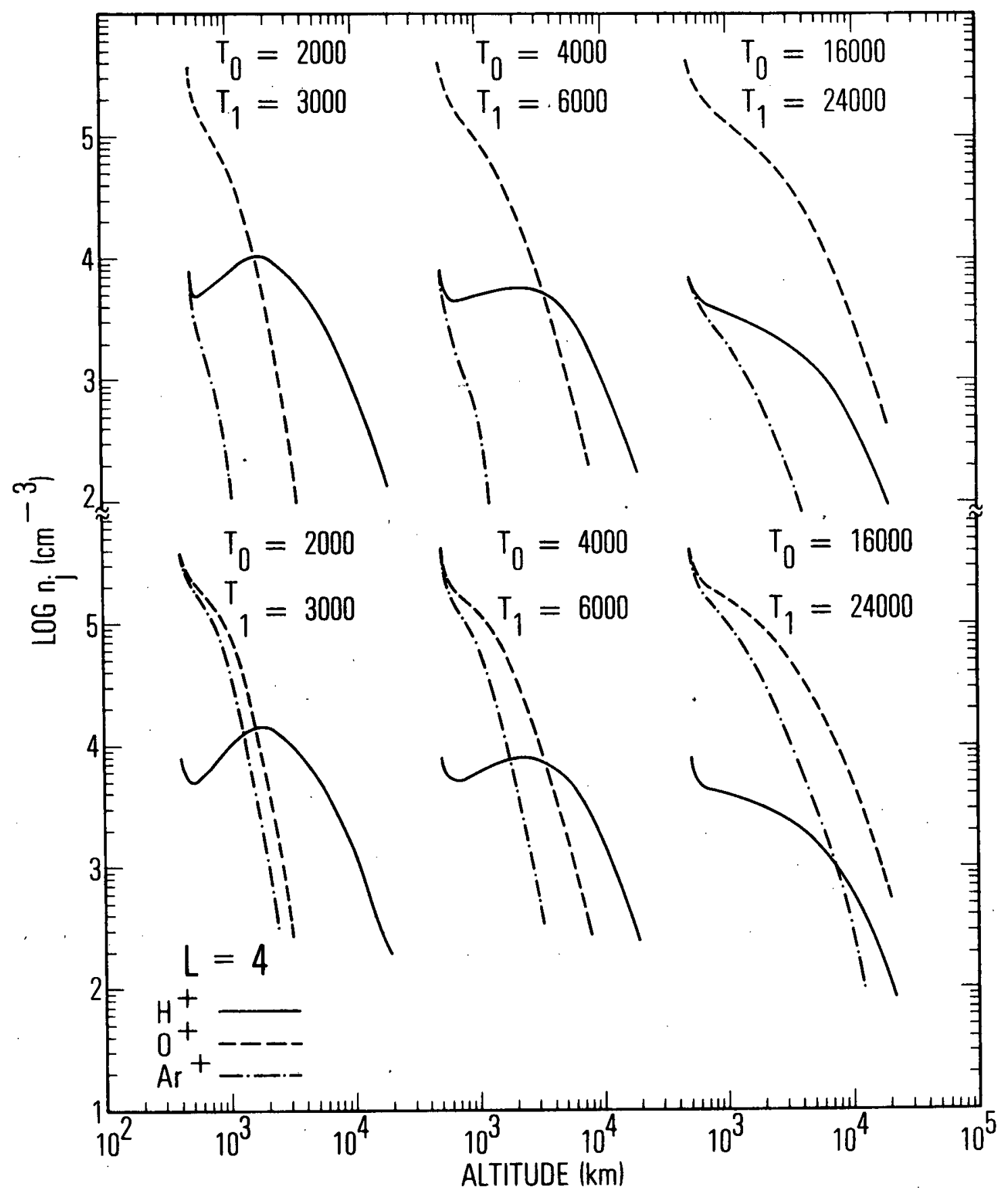

Figure 8. Calculated $L=4$ ion density distributions for various $\mathrm{Ar}^{+}$concentrations at the $500 \mathrm{~km}$ altitude boundary and several the rmal models. 
medium or by Joule heating can drastically alter the plasmasphere. At high altitudes, hydrogen can be replaced by the heavier constituents (oxygen or a combination of oxygen and argon). Some consequences of the possible changes in plasmasphere composition will be considered in the next sections. But before continuing the discussion, it should be pointed out that the calculation described above is a crude approximation to the actual situation which is very far from steady state. The argon ions will be introduced onto different $L$ shells at different times; plasma convection processes and radial diffusion can modify the spatial distribution of the argon which, because of the nature of its source, is already quite different from that of a normal plasmasphere constituent. In addition, interhemispheric flows, which may affect the plasmasphere as a whole were not included in the model. Work on including these effects is currently underway. 


\section{THIS PAGE \\ WAS INTENTIONALLY \\ LEFT BLANK}

$=$ 
V. Ring-Current and Radiätion-Belt Effects

The widespread use of ion engines in space carries certain implications for the high-energy $(E \gtrsim l 0 \mathrm{keV})$ particles that populate the earth's magnetosphere. The effects of ion engines on such high-energy populations are necessarily indirect if the injected argon ions have energies of at most $500 \mathrm{eV}$. The high-energy particles, however, are subject to the presence of plasma turbulence in the magnetospheric environment, and the level of such turbulence may possibly be enhanced or reduced by the addition of argon ions from a space engine.

It is customary, for both kinematical and dynamical reasons, to distinguish between particles having $E \sim 10-100 \mathrm{keV}$ and those having $\mathrm{E} \gtrsim 100 \mathrm{keV}$. The former are known as ring-current particles, since they account for the major share of diamagnetic disturbances observable with low-latitude magnetometers at the earth's surface. The latter are known as radiation-belt particles, since they account for most of the radiation damage to hardened spacecraft. The ring current and the radiation belts consist of electrons, protons, and heavier ions trapped in the earth's magnetic field. Such particles obey the laws of adiabatic charged-particle motion in the first approximation, and their iso-intensity profiles are toroidal in shape (e.g., White, 1966).

The ring current and radiation belts are populated with energetic particles by virtue of the dynamical interaction between the earth's magnetosphere and the solar wind. They are depleted of such particles mainly by interaction with plasma turbulence, which tends to violate the adiabatic invariants of charged-particle motion. Such violation allows particles to escape confinement by the earth's magnetic mirrox and to precipitate (i. e., 
deposit their energy) in the earth's atmosphere and ionosphere. However, the interaction of geomagnetically trapped particles with plasma turbulence is very selective, in that it is contingent upon a "resonance" between the gyration of a particle and the frequency of a wave in the turbulent spectrum (after one takes account of the Doppler shift associated with the motion of the particle through the plasma). Thus, different classes of turbulence are found to interact with different classes of particles.

Turbulence in a plasma can be created by various mechanisms. For example, the injection of a cold (i.e., mono-energetic) ion beam into a background plasma can easily lead to instability in various electrostatic wave modes (e.g., Hasegawa, 1975). The consequence of such instability is the rapid amplification of thermal noise to appreciable amplitude, such that the beam itself becomes diffused. In the case of ion engine beams, the question is whether the resulting plasma turbulence can interact resonantly with ring-current or radiation-belt particles. The answer to this question is not yet known, since beam-plasma instabilities have not yet been investigated thoroughly in this context.

Another source of instability in the magnetospheric plasma is the velocity-space anisotropy intrinsic to ring-current particle distributions. Since the magnetic mirror points of ring-current protons and electrons, are concentrated near the magnetic equator rather than being distributed uniformly along a field line, there must be relatively more energy per degree of freedom associated with particle gyration than with translation of guiding centers along field lines. This condition can easily lead to instability in certain electromagnetic wave modes. The anisotropy of 
ring-current electrons can lead to instability of a field-guided wave with right-handed polarization, i.e., the so-called "whistler" wave mode. The anisotropy of ring-current ions can lead to instability of the analogous fieldguided wave with left-handed polarization. Both instabilities require the wave frequency to be somewhat smaller than the corresponding particle gyrofrequency. Both instabilities cause velocity-space diffusion so as to reduce the anisotropy of the corresponding charged-particle species, and so as to reduce the lifetime of that species against precipitation into the earth's atmosphere (Kennel and Petschek, 1966; Cornwall, 1966; Cornwall et al., 1970). Moreover, the unstable ion-cyclotron waves generated by the anisotropy of ring-current protons are resonant with relativistic radiation-belt electrons $(E \gtrsim 2 \mathrm{MeV}$ ) and thus account for the observed precipitation of such electrons during the recovery phase of a magnetic storm (Thorne and Kennel, 1971; Vampola, 1971). It happens that the electromagnetic instabilities noted here are not effective at ring-current energies for protons outside the plasmasphere, since the larger phase velocities attained there require a correspondingly larger proton energy for cyclotron resonance. Thus, the precipitation of relativistic electrons is contingent on the spatial co-existence of ring current and plasmasphere, which occurs only during the plasmaspheric expansion characteristic of the recovery phase of a magnetic storm.

The electromagnetic proton-cyclotron instability, however, is likely to be suppressed by the presence of substantial numbers of heavy ions such as $\mathrm{Ar}^{+}$or $\mathrm{O}^{+}$in the magnetospheric plasma (Cornwall and Schulz, 1971). This means that the major mechanism for the depletion of relativistic electrons from the outer radiation belt is likely to be made 
inoperative by the widespread use of ion engines in space. The present population of such hazardous electrons is kept in balance by the occurrence of several large magnetic storms per year. Suppression of the protoncyclotron instability might, therefore, result in a major enhancement of the radiation level from relativistic electrons within a year after argonion saturation of the plasmasphere.

The enhancement of relativistic-electron radiation can be analyzed within the framework of a simple model. Consider the model equation (e.g., Schulz, 1974)

$$
\mathrm{dI} / \mathrm{dt}=\mathrm{S}-\lambda \mathrm{I},
$$

in which $I$ represents the radiation intensity, $\lambda$ the natural radiationbelt decay rate, and $S$ the strength of a weak source of relativistic electrons. The decay rate $\lambda$ is perhaps inversely proportional to the particle energy $E$, such that $1 / \lambda \approx(E / 1 \mathrm{MeV}) \times 10$ days. The solution of $(8)$ approaches the limit $I_{\infty}=S / \lambda$ as $t \rightarrow \infty$. Suppose, however, that $I$ is reduced to zero by relativistic electron precipitation (REP) events, which occur randomly in time. Thus, let $\exp (-\Delta t / \tau)$ be the probability that a time interval of length $\Delta t$ is free of $R E P$ events. The radiation intensity grows from zero in accordance with (8) after each. REP event, and so never quite reaches $I_{\infty}$. A careful implementation of this model shows that the instantaneous probability for $I$ to be in excess of some arbitrary threshold $I_{0}<I_{\infty}$ at any given time is

$$
P\left(I>I_{0}\right)=\left[1-\left(I_{0} / I_{\infty}\right)\right]^{1 / \lambda T}
$$


and that $P\left(I>I_{\infty}\right)=0$. The mean value of $I$, averaged over a time $\Delta t \gg \tau$, is given by

$$
\bar{I}=[\lambda \tau /(1+\lambda \tau)] I_{\infty}
$$

This is the natural state of affairs. The intensity of relativistic electrons in the vicinity of the plasmapause fluctuates in time in such a way that (9) and (10)are satisfied. Suppose, however, that REP events a re suppressed, e.g., by the addition of heavy ions to the plasmasphere. This environmental modification corresponds to the limit $T \rightarrow \infty$, whereupon $\bar{I}=I_{\infty}$. The mean radiation intensity has been enhanced by a factor $(1+\lambda \tau) / \lambda \tau$. Thus, if $\tau=20$ days (corresponding to the annual occurrence of about 18 REP events), the intensity of $2-\mathrm{MeV}$ electrons will be enhanced by a factor $\sim 2$ (relative to its natural mean value) by the addition of major quantities of $\mathrm{Ar}^{+}$or $\mathrm{O}^{+}$to the plasmasphere. The mean intensity of $4-\mathrm{MeV}$ electrons will be enhanced by a factor $\sim 3$. With the addition of heavy ions to the plasmasphere, the radiation intensity ceases to fluctuate between zero and $I_{\infty}$; it approaches $I_{\infty}$ as a permanent condition of the modified environment.

There is no danger that the population of ring-current protons would be similarly enhanced, since these are subject to rapid charge exchange with ambient neutral hydrogen, as a re ring -curent helium and oxygen ions on a substantially longer time scale (Tinsley, 1976; Lyons and Evans, 1976). Moreover, the addition of argon plasma (or any other type of plasma) to the natural plasmasphere would tend to enhance the loss rate 
of ring-current electrons (Brice, 1970; 1971). One should not worry about the development of a charge imbalance in the magnetosphere on account of modified precipitation rates. There is ample cold plasma in the plasmasphere to balance charges through minor modifications of the already weak ambipolar electric field, which has been included in the model calculations in the previous section.

The above estimates of $I$ and $P\left(I>I_{0}\right)$ a re based on a highly idealized model of radiation-belt dynamics. Other sources of fluctuation are quite likely to broaden the probabiliiy distribution. For example, one might expect both $\lambda$ and (especially) $S$ in (8) to depend on geomagnetic activity and therefore on time. Moreover, the magnetosphere is subject to reversible (adiabatic) compressions that change the scales of energy and distance. Such compressions (caused by slow variations of solar-wind speed) are not of dynamical interest and so are omitted from (8)-(10), but such adiabatic processes would have to be considered in formulating a model of the detailed time history of radiation dosage impacting a hardened spacecraft.

Suppression of the electromagnetic ion-cyclotron wave mode is illustrated in Figure 9. The normalized growth rate $r / \Omega_{\mathrm{p}}$ is plotted as a function of normalized wave frequency $\omega / \Omega_{\mathrm{p}}$ for selected argon/hydrogen plasma-concentration ratios. For the parameters chosen it is clear that a ratio $\mathrm{N}_{\mathrm{Ar}} / \mathrm{N}_{\mathrm{H}} \gtrsim 1$ is sufficient to suppress the proton-cyclotron branch altogether. The lower-frequency argon-cyclotron branch achieves a growth rate of at most $3 \%$ of the maximum proton-cyclotron growth rate attained in the absence of argon. Thus, the electromagnetic ion-cyclotron mode is 


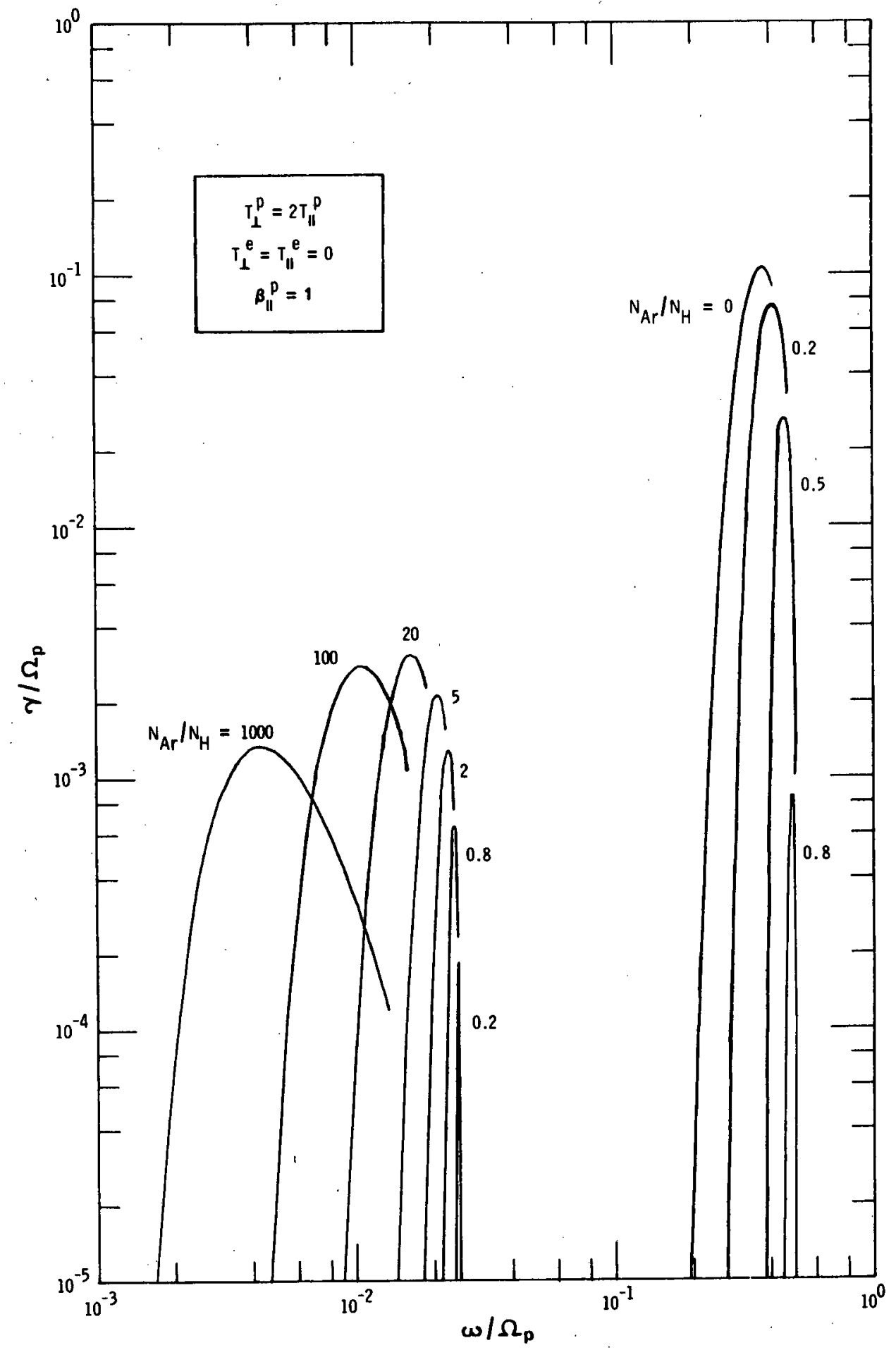

Figure 9. Lowest-order estimates for the normalized growth rate as a function of normalized wave frequency, for various relative concentrations (NAr/NH) of argon and hydrogen plasma. The method of calculation is that of Mark (1974). 
very much stabilized overall by the addition of cold argon plasma to the medium. This conclusion is consistent with the results obtained by similar methods for the addition of even lighter ions such as $\dot{\mathrm{Li}}^{+}$and $\mathrm{He}^{+}$(Märk, $1974)$.

The foregoing discussion of ring-current and radiation-belt effects might have to be modified if the injected argon ions were to have energies $\sim 3.5 \mathrm{keV}$ (Option 1 in Table I). A beam of such ions might be stopped by the mechanism of Scholer (1970) and Pilipp (1971) before the ions became geomagnetically trapped. The result would be a cold argon plasmathaving the same effects as are outlined above. On the other hand the injected 3. 5-keV ions might be scattered out of the beam by some mechanism and come to constitute a plasma having a $3.5-\mathrm{keV}$ temperature. In this latter case the injected argon plasma would contribute directly to the ring-current population, and the $\mathrm{O}^{+}$drawn up from the F-region would constitute the cold-plasma additive. The cold $\mathrm{O}^{+}$would tend to suppress the electromagnetic proton-cyclotron and helium-cyclotron instabilities of the natural ring current at frequencies below the oxygen gyrofrequency (e.g., Cornwall and Schulz, 1971). However, the hot argon plasma would become anisotropic with time because of charge exchange, and would thus constitute an additional source of free energy for instability (cf. Cornwall, 1977). The a rgon-cyclotron instability would occur at frequencies somewhat below the argon gyrofrequency. Its growth rate would be enhanced by the presence of cold $\mathrm{O}^{+}$in the plasmasphere (cf. Cornwall and Schulz, 1971). The argon-cyclotron waves would tend to resonate with relativistic electrons (cf. Thorne and Kennel, 1971) and thus to facilitate the loss of such trapped 
electrons into the atmosphere. In summary, some of the plasma-dynamical consequences of injecting hot argon plasma ( $E \geq 3.5 \mathrm{keV}$ ) may be qualitatively different from those of injecting cold argon plasma ( $\mathrm{E} \leqslant 500 \mathrm{eV}$ ). However, it is clear that in the case of cold-plasma injection by an ion engine, the intensity of relativistic-electron radiation in the earth's magnetosphere will be substantially increased. 


\section{Conclusions}

We have assessed the initial phases of the magnetospheric modification scenario of ion engine exhaust from the projected cargo orbit transfer vehicles (COTV) of the satellite power system. Aside from summarizing the emission scenarios of the various options, we have dealt with several important aspects of the initial fate of the ion engine exhaust plasma, using presently available information.

We point out at the outset that, if the critical-velocity phenomenon observed in the laboratory is applicable in the magneosphere, the problem of chemical rocket exhaust (neutral molecules) release in the magnetosphere is almost equivalent to the case of ion engine plasma release that we have analyzed because the chemical-rocket exhaust speed in the magnetosphere is likely to exceed the critical velocity for ionization of the constituents.

We have examined the question of the propagation of the ion-engine exhaust plasma beam across the geomagnetic field. We show that it is fallacious to consider the problem as a plasma beam moving across a vacuum magnetic field. The plasma beam moves across the geomagnetic field and causes a disturbance of the magnetospheric plasma in the form of an Alfvén wave. The inertia of the natural magnetospheric plasma, driven by the Alfvén wave disturbance, represents a braking force on the motion of the beam. This reaction force is able to stop the ion beam in a distance of $\sim 1000 \mathrm{~km}$, according to scaling of studies conducted for HEOS-I release of barium in the far magnetosphere. The generation of an Alfvén wave disturbance by the plasma beam further implies that an electric field of magnitude comparable to the auroral electric field but 
associated with the Alfvén wave is applied to the ionosphere at the footprint of the magnetic flux tube of the plasma beam (Figure 1). The Joule dissipation (heating) in the ionosphere by this electric field is comparable to that in the auroral region during a magnetic storm.

We have roughly modelled the density and composition changes of the plasmasphere based on the above findings that the entire ion-engine exhaust will be stopped inside the magnetosphere. It is found that the mere addition of $\mathrm{Ar}^{+}$to the plasmasphere does not drastically change the composition of the plasmasphere, but the input of heat associated with the Ar exhaust will have a tendency to change the plasmaspheric composition by allowing ionospheric $\mathrm{O}^{+}$to be forced up into the far plasmasphere where the radiation belts a re located.

We have shown that an increase in the proportion of thermal ions of high mass such as $\mathrm{Ar}^{+}$or $\mathrm{O}^{+}$can suppress the ion-cyclotron wave generation mechanism, i.e., the plasma instability associated with pitchangle anisotropy of ring-current ions. Since, in the natural state, such ion-cyclotron waves cause the loss of radiation-belt electrons by pitchangle scattering and thus limit the natural dosage level, the suppression of cyclotron-wave generation implies that the radiation belt dosage would build up to a higher level. Estimates, based on production and loss considerations applicable to the natural state of the radiation belts, indicate that ion cyclotron wave suppression may increase the radiation belt average level by as much as a factor of two or three.

Our considerations here are limited to the application of present knowledge and are limited by considerations of the initial fate of the plasma . beam exhaust. Further considerations are needed before we can clearly define the eventual fate of the $\mathrm{Ar}^{+}$exhaust plasma. 


\section{$\underline{\text { References }}$}

Alfvén, H., and G. Arrhenius, Evolution of the Solar System, National Aeronatics and Space Administration NASA SP-345, U.S. Gov't Printing Office, Washington D. C., Ch. 21, pp 383-391, 1976. Angerth, B., L. Block, U. Fahlson, and K. Sopp, Experiments with partly ionized rotating plasmas, Nucl. Fusion Supp. Part I., 39, 1962.

Brice, N., Artificial enhancement of energetic particle precipitation through cold plasma injection: A technique for seeding substorms?, J. Geophys. Res., 75, 4890, 1970.

Brice, N., Harnessing the energy in the radiation belts, J. Geophys. Res., 76, 4698, 1971 .

Byers, D. C., and V. K. Rawlin, Electron bombardment propulsion system characteristics for large space systems, Twelfth International Electric Propulsion Conference, American Institute of Aeronautics and Astronautics, Key Biscayne, Fla., Nov. 15-17, 1976.

Chiu, Y. T., J. G. Luhmann, B. K. Ching, Michael Schulz and D. J. Boucher, Jr., Magnetospheric and ionospheric impact of largescale space transportation with ion engines, Aeronautics and Astronautics, accepted for publication, 1979a; also Aerospace Technical Report SAMSO-TR-79-3, 1979a.

Chiu, Y. T., J. G. Luhmann, B. K. Ching and D. J. Boucher, Jr., An equilibrium model of plasmaspheric composition and density, $J$. Geophys. Res., 84, 909, 1979b.

Cornwall, J. M., Micropulsations and the outer radiation zone, J. Geophys. Res., 11, 2185, 1966. 
Cornwall, J. M., F. V. Coroniti, and R. M. Thorne, Turbulent loss of ring current protons, J. Geophys. Res., 75, 4699, 1970.

Cornwall, J. M., and M. Schulz, Electromagnetic ion-cyclotron instabilities in multicomponent magnetospheric plasmas, J. Geophys. Res., 76, 7791, 1971; Correction, J. Geophys. Res., 78, 6830, 1973.

Cornwall, J. M., On the role of charge exchange in generating unstable waves in the ring current, J. Geophys. Res., 82, 1188, 1977.

Curtis, S. A., and J. M. Grebowsky, Changes in the terrestrial atmosphereionosphere-magnetosphere system due to ion propulsion for solar power satellite placement, NASA Tech. Memo. 79719, Goddard Space Flight Center, unpublished, 1979.

Danielsson, 'L., and N. Brenning, Experiment on the interaction between a plasma and a neutral gas, II, Phys. Fluids, 18, 661, 1975.

Gilbody, H. B., and J. B. Hasted, Anomalies in adiabatic interpretation of charge transfer collisions, Proc. Roy. Soc., Ser. A, 238, 1956. Haerendel, G., and R. Lüst, Electric fields in the ionosphere and magnetosphere, Particles and Fields in the Magnetosphere (ed. B. M. McCormac), p. 212, Reidel, Dordrecht-Holland, 1970.

Hasegawa, A., Plasma Instabilities and Nonlinear Effects, pp. 28-43, Springer, Heidelberg, 1975.

Kauffman, H. R., Technology of electron-bombardment ion thrusters, Advances in Electronics and Electron Physics, Vol. 36, ed. L. Marton, Academic Press, New York, 1974.

Lyons, L. R., and D. S. Evans, The inconsistency between proton charge exchange and the observed ring current decay, J. Geophys. Res., 81, $6197,1976$. 
Märk, E., Growth rates of ion cyclotron instability in the magnetosphere, J. Geophys. Res., 79, 3218, 1974.

Möbius, E., R. W. Boswell, A. Piel, and D. Henry, Investigation of the critical velocity phenomenon from SPACELAB, Transact. AGU/ EOS, 59, 1162, 1979 .

Perkins, F. W., N. J. Zabusky, and J. H. Doles, III, Deformation and striation of plasma clouds in the ionosphere, 1, J. Geophys. Res., 78, 697, 1973 .

Pilipp, W. G., Expansion of an ion cloud in the earth's magnetic field, Planet. Space Sci., 19, 1095, 1971.

Scholer, M., On the motion of artificial ion clouds in the magnetosphere, Planet. Space Sci., 18, 977, 1970.

Schulz, M., Particle saturation of the outer zone: A nonlinear model, Astrophys. Space Sci., 29, 233, 1974.

Thorne, R. M., and C. F. Kennel, Relativistic electron precipitation during magnetic storm main phase, J. Geophys. Res. , 76, 4446, 1971.

Tinsley, B. A., Evidence that the recovery phase ring current consists of helium ions, J. Geophys. Res., 81, 6193, 1976.

Vampola, A. L., Electron pitch angle scattering in the outer zone during magnetically disturbed times, J. Geophys. Res., 76, 4685, 1971.

White, R. S., The earth's radiation belts, Phys. Today, $19(10), 25$, October 1966. 